\title{
Patrones de actividad y organización social de los grupos cazadores-recolectores de la cuenca del lago Salitroso, provincia de Santa Cruz (Argentina) durante el Holoceno tardío: un estudio de las modificaciones osteológicas (artropatías)
}

\section{Milena C. Morlesín" \\ Fecha de defensa: o5 de agosto de 2019 \\ Directora: Dra. Solana García Guraieb \\ Co-director: Dr. Rafael A. Goñi \\ Jurado: Dres. Leticia Cortés y Leandro Luna}

Desde una perspectiva bioarqueológica, esta tesis tiene por objetivo contribuir al conocimiento de los patrones de actividad de los grupos cazadores-recolectores de Patagonia Centro-meridional que habitaron la cuenca del lago Salitroso durante el Holoceno tardío ${ }^{1}$, a través del estudio de las modificaciones óseas de carácter patológico, tal como son las artropatías (Lieverse, Mack, Bazaliiskiy, Weber, 2016; Sofaer Derevenski, 2000). Si bien existe una multiplicidad de factores biológicos causales de tales modificaciones, -p.e. edad, sexo, herencia genética, entre otros-, es sabido que la actividad física repetida genera cambios articulares, y por ende juega un papel importante en su expresión, especialmente en determinadas articulaciones. En consecuencia, teniendo en cuenta y controlando tales factores, las artropatías son indicadores útiles para inferir patrones de actividad de las poblaciones del pasado (Bridges, 1991; Jurmain, 1977; Lieverse et al., 2016).

Las investigaciones arqueológicas en la región estuvieron orientadas a conocer los patrones de movilidad y uso del espacio de los cazadores-recolectores y su vinculación con los cambios climáticos y ecológicos registrados en Patagonia durante el Holoceno, particularmente en el tardío (Goñi, 2010; Goñi y Barrientos, 2004; Goñi, Barrientos y Cassiodoro, 2000-2002). En este sentido, Goñi y colaboradores han postulado un modelo de poblamiento regional que sostiene que la disponibilidad de espacios para la ocupación, la dinámica de movilidad y los sistemas organizativos de los grupos locales se vieron fuertemente influenciados por las fluctuaciones ambientales experimentadas en Patagonia durante el Holoceno tardío (ca. 2500-últimos 1000 años AP sensu Stine, 1994) (Goñi, 2010; Goñi y Barrientos, 2004; Goñi et al., 2000-2002).

* Instituto Nacional de Antropología y Pensamiento Latinoamericano (INAPL) - CONICET. 3 de febrero 1378 (CP C1426BJN) Ciudad Autónoma de Buenos Aires. E-mail: mile.morlesin@gmail.com

${ }^{1}$ Se analizará del Holoceno tardío el rango cronológico de los 2600 a los 350 años AP.
Durante este período, se registra una tendencia hacia una humedad ambiental decreciente, con condiciones de mayor aridez, cuyo punto máximo fue alcanzado durante el fenómeno climático conocido como la Anomalía Climática Medieval (ACM) (ca. 1200 a 600 años AP, 800 a 1350 años cal.) (Stine, 1994). En los picos de mayor aridez, la presencia de agua en el ambiente habría quedado circunscripta a loci puntuales, con disponibilidad de recursos críticos (leña, vegetales, animales, reparo) que habrían tenido el potencial de actuar como concentradores de poblaciones. Estas nuevas condiciones ambientales habrían generado cambios en los sistemas organizativos de los grupos locales, orientados a la reducción de la movilidad residencial, al constreñimiento espacial, al nucleamiento de poblaciones, y a la ampliación de los rangos de acción en materia de movimientos logísticos (Goñi, 2010; Goñi y Barrientos, 2004; Goñi et al., 2000-2002).

En relación con el modelo de poblamiento en el cual se enmarca esta tesis, y dado que entre los factores etiológicos de las artropatías se encuentra el uso del cuerpo y la actividad física repetida, su estudio se presenta como una vía para evaluar, en clave temporal, los niveles de actividad y stress mecánico. A su vez, se busca discutir la existencia de cambios en la organización de las actividades y tipos de tareas realizadas, en relación con el proceso de reducción de la movilidad residencial y nucleamiento poblacional en la cuenca para el último milenio (Goñi, 2010; Goñi et al., 2000-2002).

Los cambios patológicos en las articulaciones son una fuente de información relevante, ya que el individuo a lo largo de su vida experimenta modificaciones de su estructura ósea (desgaste articular, fracturas, microtraumas, entre otros) (Hawkey y Merbs, 1995). Dichas modificaciones presentan una distribución puntual que puede ser leída como una marca de actividad o stress mecánico. Cabe destacar, que no todas las actividades realizadas dejan evidencias observables en el esqueleto, y que distintas actividades pueden dejar la misma señal ósea (Sofaer Derevenski, 2000). A su vez, las actividades realizadas a lo largo de la vida de los individuos responden a tradiciones en contextos sociales y temporales particulares, a la vez que dan cuenta de las diferencias sociales existentes entre ellos (Porčić y Stefanović, 2009). De esta forma, si 
la organización social del grupo para realizar las tareas diarias se basa en un sistema de asignación de actividades particulares a cada sexo y/o grupo etario (Hooper, Demps, Gurven, Gerkey y Kaplan, 2015), y/o si dicha organización y actividades varían a lo largo del tiempo, las alteraciones del tejido óseo también expresarán dichas diferencias. Estudiar las artropatías permite conocer su manifestación en la población bajo estudio, y aproximarnos a la dimensión individual de las actividades. Bajo este marco, los objetivos particulares de este trabajo son:

» Caracterizar la muestra de acuerdo a la presencia, naturaleza, distribución e intensidad de las artropatías de los individuos adultos de ambos sexos que integran la colección osteológica de la cuenca del lago Salitroso.

》 Evaluar la relación entre los patrones de variación de las artropatías, particularmente de la Osteoartrosis (OA), con aspectos fisiológicos básicos como el sexo y la edad.

» Analizar la potencial existencia de una relación entre las manifestaciones osteoartrósicas y los comportamientos humanos vinculados al uso del cuerpo y a la ejecución de las tareas cotidianas, a partir de:

A. La presencia de OA en la columna vertebral, en el hombro, la pelvis y la rodilla.

B. La presencia de asimetría bilateral en la manifestación de la OA en las articulaciones apendiculares.

Para ello, se analizó una muestra compuesta por 14 individuos de ambos sexos, adultos (mayores a 20 años, dado que representa el umbral mínimo de edad para abordar el estudio de rasgos óseos degenerativos), integrantes de la colección osteológica recuperada en la cuenca ${ }^{2}$. Los restos fueron hallados en estructuras de entierro de tres modalidades distintas: entierros bajo bloque (EBB), nichos y chenques (García Guraieb, 2010; García Guraieb, Goñi, Tessone, 2015). Asimismo, los individuos analizados presentan una cronología que abarca el período entre ca. 2600 y los 350 años AP, y fueron agrupados en dos bloques temporales de acuerdo a la ACM: uno cuya cronología es previa a la ACM (2600-1200 AP) y otro posterior a la ACM (ca. 800-350 años AP).

Se relevaron macroscópicamente 1598 superficies articulares vertebrales y 182 superficies articulares apendiculares, a los fines de realizar el diagnóstico diferencial de diversas artropatías tales como los nódulos de Schmorl, Síndrome de Reiter, Artitis reumatoidea, Artritis psoriasica, Espondilitis anquilosante, Hiperostosis esquelética idiopática difusa (DISH) y OA. Se relevaron las

${ }^{2}$ La colección cuenta hasta el momento con aproximadamente 97 individuos de ambos sexos y de todas las edades, desde perinatos hasta adultos mayores (para mayor detalle ver García Guraieb, 2010). lesiones óseas proliferativas (remodelación del margen de la carilla articular vertebral, labiaciones o lipping, osteofitos y eburnación) (Arrieta y Mendonça, 2011; Luna, Aranda y Amorim Alves, 2017; Rogers y Waldron, 1995; Rogers, Waldron, Dieppe y Watt, 1987; Sofaer Derevenski, 2000; Waldron, 2009) y las alteraciones inflamatorias (porosidad o pitting) (Rojas-Sepúlveda, Ardagna y Dutour, 2008; Sofaer Derevenski, 2000). En el caso de la OA, su diagnóstico positivo se realizó a través de dos criterios: a) articulaciones con signos de eburnación, y/o b) articulaciones con dos o más de las siguientes lesiones: remodelación del margen de la carilla articular, labiado u osteofitos (Rogers y Waldron, 1995; Waldron, 2009), y se asignó un grado de severidad a la OA (Neves, 1984; Scabuzzo, 2010). Por último, se realizó el cálculo de frecuencia, severidad y distribución sexo-etaria y temporal de los casos positivos de OA. Se evaluaron las diferencias estadísticas existentes en la expresión de las artropatías en las articulaciones vertebrales y apendiculares entre los grupos de edad, sexo, bloque temporal y lateralidad mediante la Prueba Exacta de Fisher y $\mathrm{Chi}^{2}$, con un nivel de significación de $\mathrm{p}<0,05$. Los datos obtenidos fueron procesados con el paquete informático PAST 3.20.

Los resultados obtenidos indican que todos los individuos exhiben OA. A nivel de la columna vertebral, la región lumbar y el segmento inferior de la región torácica exhiben las mayores frecuencias de OA. En el caso de las articulaciones apendiculares, la pelvis es la que exhibe la mayor frecuencia de OA, seguida de las articulaciones del hombro y la rodilla. En relación con el sexo, se hallaron frecuencias similares de OA entre ambos para todas las articulaciones bajo estudio. En lo que respecta a la edad, los individuos mayores a 40 años presentan mayores frecuencias de afectación. A su vez, se obtuvo una mayor frecuencia de casos positivos de OA en los individuos de momentos previos a la ACM a nivel de la columna vertebral. Por último, de acuerdo a la lateralidad de las articulaciones apendiculares, el lado izquierdo exhibe mayores frecuencias de OA.

En términos generales, los casos de $\mathrm{OA}$ indican bajos niveles de exigencia articular. La ausencia de diferencias estadísticamente significativas de la OA en relación con el sexo, podría indicar que, ante una factible división de tareas entre estos, los individuos femeninos y masculinos habrían realizado sus actividades diarias comprometiendo diversos sistemas articulares con niveles de exigencia similares (Lieverse et al., 2016; Sofaer Derevenski, 2000). Asimismo, la mayor frecuencia de $\mathrm{OA}$ en individuos mayores a 40 años, implica que, en esta instancia, no puede descartarse que el patrón observado responda al carácter acumulativo del desgaste fisiológico de las articulaciones, y a la intervención de procesos sistémicos asociados al envejecimiento, sumado a la sobrecarga mecánica en las articulaciones. No obstante, el hallazgo 
de individuos menores a 40 años con casos positivos de OA dan cuenta de la existencia de niveles de actividad física y estrés mecánico desencadenantes del desarrollo de la OA en la población joven. A su vez, la existencia de diferencias estadísticamente significativas entre los dos bloques temporales, puede estar asociada a la mayor demanda mecánica a la que estuvieron expuestos los individuos durante el Holoceno tardío inicial, asociado a la carga y traslado de pesos sobre la columna vertebral durante los movimientos residenciales.

En relación con la bilateralidad, el lado izquierdo presenta una mayor frecuencia de OA. No se hallaron diferencias estadísticamente significativas $(p=0,056)$. Sin embargo, el valor obtenido es cercano a la significación estadística. De esta forma, no puede rechazarse la suposición de una predisposición hacia el mayor uso del lado izquierdo, la cual puede responder a que este lado es utilizado para la carga de pesos sobre miembros superiores, lo que a su vez ocasiona un mayor esfuerzo sobre ese lateral en los miembros inferiores. Asimismo, se plantea que el lado izquierdo suele ser usado para aplicar fuerza y dar impulso al movimiento (Palmer, 2012).

A modo de síntesis, a partir de la distribución de la $\mathrm{OA}$, se propone que la actividad física repetida habría sido una de las causantes de su manifestación. Dichas actividades habrían consistido en la carga y traslados de pesos a lo largo de extensas porciones del terreno, tal como el acarreo de personas (p. ej. niños sin capacidad de trasladarse por sus propios medios), de recursos animales y objetos pesados (p. ej. cueros, agua, leña, recursos animales y vegetales, materias primas líticas, entre otros) sobre el sector del cuello/hombro y la espalda, afectando principalmente al segmento inferior de la región torácica y a la región lumbar (Bridges, 1991). Por último, la presencia de lesiones traumáticas (p. ej. Espondilolísis de la quinta vértebra lumbar) y malformaciones congénitas ( $p$. ej. presencia de sextas vértebras lumbares, malformaciones en la región cervical) (García Guraieb, 2010) pueden favorecer la presencia de lesiones osteoartrósicas, ya que alteran las curvaturas normales de la columna, reforzando así la hipótesis de la existencia de procesos de estrés mecánico.

\section{Referencias citadas}

»Arrieta, M. y Mendonça, O. (2011). Enfermedad degenerativa articular y uso del cuerpo en Rincón Chico 21. Revista Argentina de Antropología Biológica, 13, 03-17.

" Bridges, P. (1991). Degenerative joint disease in huntergatherers and agriculturalists from the southeastern USA. American Journal of Physical Anthropology, 85, 379391.
» García Guraieb, S. (2010). Bioarqueología de CazadoresRecolectores del Holoceno Tardío de la Cuenca del lago Salitroso (Santa Cruz): Aspectos Paleopatológicos y Paleodemográficos. (Tesis Doctoral inédita), Universidad de Buenos Aires, Argentina.

"García Guraieb, S., Goñi, R. y Tessone, A. (2015). Paleodemography of late Holocene hunter-gatherers from Patagonia (Santa Cruz, Argentina): an approach using multiple archaeological and bioarchaeological indicators. Quaternary International, 365, 147-158.

» Goñi, R. (2010). Cambio Climático y Poblamiento Humano durante el Holoceno tardío en Patagonia Meridional. Una Perspectiva Arqueológica. (Tesis Doctoral inédita), Universidad de Buenos Aires, Argentina.

» Goñi, R. y Barrientos, G. (2004). Poblamiento tardío y movilidad en la cuenca del lago Salitroso. En T. Civalero, P. Fernández, y G. Guraieb (Eds.), Contra Viento y Marea. Arqueología de Patagonia (pp. 313-324). Buenos Aires: Instituto Nacional de Antropología y Pensamiento Latinoamericano -Sociedad Argentina de Antropología.

» Goñi R., Barrientos, G. y Cassiodoro, G. (2000-2002). Condiciones previas a la extinción de las poblaciones humanas del sur de Patagonia: una discusión a partir del análisis del registro arqueológico de la cuenca del Lago Salitroso. Cuadernos del Instituto Nacional de Antropología Pensamiento Latinoamericano, 19, 249-266.

» Hawkey, D. y Merbs, C. (1995). Activity induced musculoskeletal stress markers (MSM) and subsistence strategy changes among ancient Hudson Bay Eskimos. International Journal of Osteoarchaeology, 5(4), 324-338.

» Hooper, L., Demps, K.., Gurven, M., Gerkey, D. y Kaplan, H. (2015). Skills, division of labour and economies of scale among Amazonian hunters and South Indian honey collectors. Philosophical Transaction Royal Society, 370, 1-11.

» Jurmain, R. (1977). Stress and the etiology of osteoarthritis. American Journal of Physical Anthropology, 46, 353-366.

» Lieverse A., Mack, B., Bazaliiskiy, V. y Weber, A. (2016). Revisiting osteoarthritis in the Cis-Baikal: understanding behavioral variability and adaptation among middle Holocene foragers. Quaternary International, 405, 160-171.

»Luna, L., Aranda, C. y Amorim Alves, A. (2017). Reflexiones sobre el relevamiento y análisis comparativo de patologías osteoarticulares en restos esqueletales humanos. Revista Argentina de Antropología Biológica, 19(1), 1-8

» Neves, W. (1984). Estilo de vida e osteobiografía: a reconstituiçao do comportamento pelos ossos 
humanos. Revista de Pré-historia, VI, 287-291.

"Palmer, J. (2012). Busy bones. Osteoarthritis and Musculoskeletal Markers as Evidence of Physical Activity and Social Differentiation in post-medieval the Netherlands. (Tesis de Master inédita). Universiteit Leiden, Holanda.

»Porčić, M. yStefanović, S. (2009). Physical activity and social status in Early Bronze Age society: The Mokrin necropolis. Journal of Anthropological Archaeology, 28(3), 259-273.

" Rogers, J. y Waldron, T. (1995). A field guide to joint Disease in Archaeology. Chichester: John Wiley \& Sons.

" Rogers, J., Waldron, T., Dieppe, P. y Watt, I. (1987). Arthropathies in palaeopathology: The basis of classification according to most probable cause. Journal of Archaeological Science, 14, 179-193.
»Rojas-Sepúlveda, C., Ardagna, Y. y Dutour, O. (2008). Paleoepidemiology of vertebral degenerative disease in pre-columbian Muisca series from Colombia. American Journal of Physical Anthropology, 35, 416-430.

"Scabuzzo, C. (2010). Actividad, Patología y Nutrición de los Cazadores-Recolectores Pampeanos. (Tesis Doctoral inédita), Universidad Nacional de La Plata, Argentina.

»Sofaer Derevenski, J. (2000). Sex differences in activityrelated osseous change. American Journal of Physical Anthropology, 111, 333-354.

"Stine, S. (1994). Extreme and persistent drought in California and Patagonia during mediaeval time. Nature, 369, 546-549.

»Waldron, T. (2009). Paleopathology. Cambridge: Cambridge University Press. 